\title{
Human Oral Processing and Texture Profile Analysis Parameters - Bridging the gap between the sensory evaluation and the instrumental measurements
}

\begin{abstract}
Studies in food oral processing are becoming increasingly important with the advent of the aged society. The food oral processing model of Hutchings and Lillford (J Texture Studies 19 103-115) describes the structural breakdown and lubrication of ingested food before the swallowing stage, and has been revisited in the present paper. The instrumental technique Texture Profile Analysis (TPA) purports to mimic the first two bites of mastication and its ease of use has lured some researchers to use it without a critical eye.

In this paper we consider inconsistencies in the Hutchings and Lillford model with the hope that it might be further refined. With regard to TPA we question the validity of the data generated and urge authors caution before they publish results from the test protocol. If results are published then the $x$-axis should be viewed as deformation or strain, and not time. Hardness should be represented by the breaking stress. Adhesiveness should be measured at a medium strain taking into account the surface properties of the plunger. The ratio of the energy estimated by the area under the curve obtained from the second and the first bites $\left(A_{2} / A_{1}\right)$ should be called recoverability and not cohesiveness.
\end{abstract}

\section{Practical applications}

To select the suitable foods for disadvantaged people, a simple and easy method to judge the safety of each food is required. Correct usage of TPA is necessary. The users of TPA should understand the limitation and validity of TPA. While hardness and related parameters of TPA can be used, other methods should be used to get adhesiveness and cohesiveness.

\section{Introduction}

Aspiration induced pneumonia has become a serious problem in our ageing society and many studies have been undertaken to prevent it. Many recent food 
oral processing studies start by accepting the breakdown path model of Hutchings and Lillford (1988) which schematically represents the trajectory of foods during oral processing. According to this model, weakly structured foods such as juice or raw oysters may be swallowed immediately. If it has a stronger structure such as meat or biscuit it must be broken down and mixed with saliva. When the structure is weak and the moisture content low (as in sponge cake), then it needs to absorb saliva to form a bolus.

Chen (2009) systematically reviewed food oral processing trying to combine the oral physiology, food physics, psychology and related disciplines. He also initiated and convened the international conferences on food oral processing (Chen and Engelen, 2012; Chen and Loret, 2017) which are now organized every two years, raising the profile of this important area of research.

To date publications on the structure and texture during the breakdown of foods have been heavily influenced by two ideas:

1. The breakdown path model devised by Hutchings and Lillford (1988) which proposes that for a food to be swallowed, two criteria must be reached. Firstly its structure must be broken down till it reaches a threshold and secondly it must become lubricated until a second threshold is achieved. The oral processing that is required to meet these two thresholds is commonly referred to as the oral trajectory. These thresholds are illustrated in figure 1 and the intersection of the threshold planes forms a bar within which the food can be swallowed.

2. The second idea is that we can use an instrument to mimic what goes on in the mouth, and from the first two bites alone, identify many of the textural characteristics of the food. This idea originates from the protocol described by Friedman, Whitney and Szczesniak (1963) which is normally referred to as Texture Profile Analysis (TPA). Its popularity is perhaps because it is simple to carry out and that it lures the operator into believing that as it mimics biting, and that it might provide measures of food texture analogous to those experienced during oral processing.

In this paper, the past and present discussion around the oral processing model of Hutchings and Lillford is considered in relation to the validity of the parameters of TPA, which attempts to mimic the initial steps (first two bites) of the same breakdown process. 


\section{Hutching and Lillford model of food oral processing}

\section{I-1. Structure breakdown}

In the dentistry, the particle size distribution after mastication has been studied quantitatively as an index of masticatory performance (Olthoff, van der Bilt, Bosman and Ueizen, 1984; van der Bilt, Olthoff, van der Glas and van der Weelen, 1987). Their studies were based on the fracture mechanics which explain mathematically the disintegration of food, though originally used to understand the grinding of rocks, coals and such hard materials. Jalabert-Malbos, MishellanyDutour, Woda, \& Peyron (2007) measured the particle size distribution of bolus formed from 10 natural foods including nuts, carrots, ham and cheese, just before swallowing they found that most foods are degraded into smaller particles $(<2 \mathrm{~mm})$. Larger particles in bolus arise from softer foods such as gherkins and green olives. They attributed the size difference of particles in boli formed from hard and soft foods to the following physiological reason: softer particles are not liable to injure the upper digestive mucosae. It should be remembered that softer and round particles are sensed as less gritty than hard and anisotropic irregular shape particles with a similar size (Tyle, 1993; Engelen, van der Bilt, Schipper and Bosman, 2005). More recently, Chen, Khandelwal, Liu and Funami (2013) examined the relation between the particle size and the hardness of foods and found that bolus particle size decreased with the increase of food hardness corroborating the previous finding of Jalabert-Malbos et al (2007). Therefore, although the particle size is an important criterion for safe swallowing, and is a good measure for the structural breakdown, it is not possible to find a single particle size below which the swallowing is triggered.

Prinz and Lucas (1997) studied the food oral processing using Brazil nuts and raw carrots. Although these two foods have very different mechanical properties, they are found to be swallowed after approximately the same number of chewing cycles at which point the cohesive force peaked for both foods. They concluded that the swallowing time is determined by the maximum cohesiveness, and beyond that time excessive saliva floods the bolus making it less cohesive. They calculated the cohesive force $=F_{V}-F_{A}$, where $F_{V}$ is a viscous force, and $F_{A}$ is an adhesive force (assuming that the particles in bolus are spherical).

Chen and Lolivret (2011) questioned the optimum swallow model of Prinz and 
Lucas (1997) because if the bolus has the maximum cohesiveness at the swallow point, then the effort to swallow may be higher, and therefore it is more logical to assume that the flowability is the key determinant of the swallow point. Chen and Lolivret (2011) examined the flowability and oral residence time for 28 fluid foods (yogurt drink, mayonnaise, strawberry jam, etc). They found that peanut paste (peanut butter) showed the second longest oral residence time $7 \pm 1.7 \mathrm{~s}$ (after $7.7 \pm 1.2 \mathrm{~s}$ of honey) and the highest perceived difficulty of swallowing. They found a positive correlation between the shear viscosity and the perceived difficulty of swallowing. The maximum force and the work required to stretch a fluid bolus were shown highly correlated with the oral residence time and the ease of swallowing. From these experimental results, Chen and Lolivret (2011) hypothesized that the flowability and stetchability were relevant in determining the trigger of swallowing. They thought that as the bolus becomes more flowable and stretchable, and once Prinz and Lucas's maximum cohesiveness is obtained, then less effort is required to initiate the swallow. However, they did not seem concerned that excessive chewing might induce flooding of the bolus (Prinz and Lucas,1997). This point should be further studied in relation with the risk of aspiration by flooded boli.

Rosenthal and Share (2010) studied the oral processing of peanut, peanut meal (crushed into small pieces $(0.5-2 \mathrm{~mm})$, and peanut paste (further milled to smooth texture) in relation to Hutchings and Lillford's model. Unsurprisingly both the time to the first swallow and clearance decreased as the degree of the structural destruction progressed, longest for whole peanut and shortest for peanut paste. Temporal Dominance of Sensations (TDS) showed the five sensations (Hard $\rightarrow$ Crunchy $\rightarrow$ Chewy $\rightarrow$ Soft $\rightarrow$ Compacted on teeth) for whole peanut as time progressed, while only three sensations occurred for peanut meal (Chewy $\rightarrow$ Soft $\rightarrow$ Compacted on teeth) and in the case of peanut paste only two sensations (Soft $\rightarrow$ Sticks to palate) existed. It is not surprising to notice that comminuted foods lose their 'hardness' and 'crunchiness' sensations, but Rosenthal and Share (2010) considered it odd that in the case of peanut paste, 'sticks to palate' increases with the time. These authors observed a similar behaviour with other oils seed pastes (Hawthornthwaite, Ramjan and Rosenthal, 2015) and they coined the phrase "hard to swallow".

Using sesame paste, Rosenthal and Yilmaz (2015) tried to understand the mechanism underlying this behaviour. They observed the physical properties (compressive and adhesive forces) of sesame paste while adding water and measuring water activity. The initial water activity ( $A w)$ of the sesame paste was 
0.58 , and this value barely changed until $4 \%$ water addition, there was then a gradual rise reaching an Aw of 0.90 at about $12 \%$ added water, at which point it ceased to behave as a liquid and showed the first (of two) peaks in adhesiveness. Compressive force peaked at $25 \%$ added water which also corresponded to the second and larger peak in adhesiveness. Further addition of water resulted in a gradual increase in Aw which leveled off at 0.99 (28\% added water). At 35\% added water it no longer behaved as a solid. The authors interpreted the increase of compressive and adhesive forces as the absorption of added water to cell debris resulting in a hard, sticky mass. The cell debris was originally suspended in oil, but on hydration eventually forms an oil in water emulsion. Only when it is diluted by about one third does it start lose its solidity as the emulsion is progressively thinned by the addition of water.

\section{Figure 1}

The 'hard to swallow' concept does not deny the effectiveness of Hutchings and Lillford's oral trajectory model, but contributes to its refinement. The left hand image of figure 1 is the published trajectory for hard to swallow peanut paste (Rosenthal \& Yilmaz, 2015) appears to move towards negative time. Obviously this is impossible and is actually a distortion resulting from perspective of three dimensional images represented in two dimension. The right hand image of figure 1 above is the revised trajectory redrawn on Hutchings and Lillford's model that has been rotated through $90^{\circ}$ and which overcomes this distortion. In such a modified schematic, the time monotonically increases (towards the reader) while the degree of structure initially increases simultaneously with a decrease in the degree of lubrication. However with further secretion of saliva there is a gradual increase in lubrication and a loss of structure towards the final swallow. This is possible if we recall Hiiemae and Palmer's process model (Hiiemae, 2004) for feeding where the processing cycles are repeated until the second "transport ?" question (the judgement for the bolus to go to the next step) can be answered in the affirmative. When sesame paste sticks to the tongue and palate, the question in Hiiemae and Palmer's model cannot be answered affirmatively until the time passes so that the paste absorbs more water. During this short time, the "hard to swallow" sensation can occur. Clearly time can only increase, but in the food examples mentioned above, Hutchings and Lillford's concepts of lubrication and degree of structure can both decrease and increase, during the oral processing. An increase in the structure coordinate of the trajectory can be easily imagined with the absorption of water (or saliva) by powdery foods (e.g. Rosenthal \& Pang, 2018). It is well-known that the flowability of powders decreases with increasing 
moisture content. Yet further increase in moisture content surely increase the flowability (Juarez-Enriquez, et al., 2017). Here, we should also think about the relation between the flowability and lubrication. In the example of powders, the increase in the moisture content leads to a decreases in the flowability, while a further increase in the moisture content increases the flowability through the creation of a slurry. In this latter case, the lubrication in the sense of Hutchings and Lillford increases. In other words Hutchings and Lillford's lubrication is similar to the flowability of powders after the absorption of high levels of moisture. Therefore, it should be noticed that the structure axis and the lubrication axis in the Hutchings and Lillford are not independent, but the structure and the lubrication are correlated each other as shown in the above example of powders.

An essential point of Figure 1 (right) is that the degree of lubrication initially decreases while the degree of structure increases, and then the degree of lubrication increases while the degree of structure decreases. The peanut and sesame pastes studied by Rosenthal and co-workers, exhibited a "hard to swallow" sensation resulting from the addition of saliva or water. Is this phenomenon limited to foods with comparatively low water content? Polymers of animal or plant origin such as collagen and amylose (main ingredient of starch, Nishinari and Fukada, 1980) are known to become firmer with increasing water content, which is related to the increase in the crystallinity. Some dried foods such as dried bonito, laverbread, dried soybean powders etc are usually eaten after adding water or together with moistened foods, but sometimes tasted without adding water. In such a situation, it is expected that the hardness would increase first to some extent and then decrease with increasing saliva content. If this is not detected by humans it is not called texture. As Bourne says (2000), physical properties which are not detected by human senses are not textural properties. Therefore, if the humans do not detect the hardness change in this phenomenon, it is not a textural property although it can be detected and explained by physics.

Here, another question arises. To what extent are human beings sensitive and can the threshold value be changed by training? Tyle (1983) and Engelen et al (2005) found that the minimum size of particles which humans can detect as gritty, depends on the hardness and the shape of the particles. If the panelists are trained, do they detect more subtle difference in the grittiness? This problem of the threshold concerns all textural attributes, grittinesss, hardness, adhesiveness, etc.

As Bourne (2000) says, instruments measure only one aspect of the texture 
which is a multi-faceted. Recently, many masticating machines have been created trying to analyze the texture as a whole (Peyron and Woda, 2016), and if the motion of such instruments faithfully mimic human mastication, then analysis of mastication become possible, in comparison the simple uniaxial compression employed in TPA seems wholly insufficient for such a purpose. However, no systematic analysis of mimicking mastication has yet been carried out.

\section{I-2. Lubrication}

There have been many papers to clarify the effect of structure breakdown and increasing lubrication in the oral processing. Pereira, de Wijk, Gavião and van der Bilt (2006) showed that tap water or alpha-amylase solution reduced the jaw movement and muscle activity for dry foods (Melba toast, cake), but it did not influence the chewing of fatty (cheese) and wet products (carrot). Thus, the addition of fluids facilitated chewing, and enhanced the structure breakdown and lubrication. Pereira, Gavião, Engelen and van der Bilt (2007) demonstrated that the addition of fluid could significantly reduce the number of chewing cycles for some dry foods because of enhanced bolus flowability in the presence of extra fluid. As with the hard-to-swallow oil seed pastes, if we bypass the initial structural breakdown and focus on the secondary oral processing characteristics, then hydration becomes the dominant factor in the oral processing of dry, carbohydrate rich foods such as crackers and Melba toast (Rosenthal and Pang 2018). Engelen, Fontijn-Tekamp and van der Bilt (2005) demonstrated that butter reduced the chewing cycles for dried foods such as toast and cake. More recently van Eck and co-workers (2018) reported that toppings such as cheese spread, mayonnaise on the bread reduced the chewing effort by enhancing the lubrication.

Hutchings and Lillford (1988) recognized that lubrication could arise from both hydration and/or oil. Recently tribological measurements have been carried out (Chen and Stokes, 2012; Stokes , 2012; Stokes, Boehm and Baier, 2013; Torres, Andablo-Reyes, Murray and Sarkar, 2018). In the food oral processing, bulk rheological properties detected by first bite, chewing (comminution), deformation, granulation dominate the sensation in the initial stages. During the later stages of mastication and incorporation of saliva result in formation of a bolus. Prior to swallowing the tribological sensation between bolus and tongue/palate become dominant. This is caused by the length scale change from the order of centimeters for ingested food to the order of micrometers in the case of thin 
layers between bolus and tongue/palate. Most tribological measurements were done using a mini-traction machine which enables detection of the frictional force of food layers between the rotating ball and the elastomeric (usually polydimethyl siloxan) disk (Stokes, 2012). Chojnicka-Paszun, de Jongh and de Kruif (2012) found a good correlation between the perceived creaminess and the friction coefficient observed by a mini-traction machine (Figure 2 ).

Figure 2

It is widely accepted that the perceived intensity in the sensory evaluation is correlated with the instrumentally observed quantity by a power law equation. A good fitting by a straight line shown in the Figure 2 suggests that the exponent is close to one, indicating that the creaminess is almost linearly correlated with friction coefficient. Since creaminess has been a difficult sensation to measure instrumentally this is a very encouraging result. However, the geometry used for most tribological measurements is still limited to hard surfaces (metal, glasses, Teflon, etc), and thus Dresselhuis, de Hoog, Cohen Stuart and van Aken (2008) used a pig's tongue as a substrate to assess lubrication effects of food emulsions to better mimic the real situation in the mouth (Stokes, 2012). Further development in instrumentation are expected. An artificial tongue was used to get a better understanding of the squeezing of soft food between the tongue and the palate (Ishihara, et al. 2014).

In the case of some foods such as noodles, the structure doesn't need to be broken down, and the slippery strands are swallowed without chewing. Lubrication related characteristics are related to frictional properties. The friction force between noodle strands were measured by pulling noodles glued to a holding plate attached to a plunger, which is in contact with another noodle strand glued to it. The slipperiness, the inverse of the frictional force, of rice noodle strands was found to increase with increasing concentration of added alginate or pectin to rice flour (Nitta et al, 2018). This appreciation of slippery sensations in food is related to culture. In Western countries, slurping foods is not considered good manners while in Asian countries people enjoy noodles in such a way where the structure is not so much broken down and swallowed because of the slippery and already lubricated characteristics (when covered with soup).

\section{Parameters of Instrumental Texture Profile Analysis}


In food science and technology area, the most frequently used method to obtain the relation between the sensory evaluation and the instrumental measurement is TPA which was proposed and promoted by Szczesniak and Bourne (Szczesniak, 2002; Bourne 2002). The technique is a two-bite (double compression) type test from which various parameters (named: hardness, adhesiveness, cohesiveness, brittleness/fracturability, elasticity/springiness, gumminess, chewiness) can be obtained. The reason why this method has been so often used is its simplicity. Most researchers use some kind of texture analyzer, originally the General Food Texturometer (GFT) was used, though this has now been replaced by uniaxial machines (Fiszman and Tarrega, 2017; Nishinari and Fang, 2018). Unfortunately, many papers lack important information or misinterpret the TPA parameters. The discussion of the TPA parameters in the present paper is not exhaustive because the above mentioned books and review papers have already described these systematically. In this short paper, the validity of TPA parameters especially hardness, adhesiveness and cohesiveness is discussed.

TPA is a testing protocol which has evolved over time. It was originally developed by workers at General Foods (Friedman, Whitney and Szczesnizk 1963) who had adapted the MIT denturometer (Proctor, Davison et al. 1955, Brody 1957) to create the General Foods Texturometer (GFT). Yet over the years the technique evolved notably by Bourne (1968) who adapted the protocol to operate on an Instron Universal Testing Machine in which forces are applied normal to the sample instead of the arctuate action of the GFT. In addition to changing the texturometer, he replaced the plunger of fixed contact area with a platen to squash the sample and the rate of compression was greatly reduced from an average speed $17.78 \mathrm{~mm} . \mathrm{s}^{-1}$ to just $0.83 \mathrm{~mm} . \mathrm{s}^{-1}$. Since Bourne's modification, the protocol has been adapted by numerous workers, so much so that the only thing that seems to have remained as a constant is that it is a two-bite test! Some examples which show the extent of the variation in the test protocol are:

- The speed of compression ranging from $0.02 \mathrm{~mm} \cdot \mathrm{s}^{-1}$ (Ravi and Susheelamma 2004) to $100 \mathrm{~mm} \cdot \mathrm{s}^{-1}$ (Allais, Edoura-Gaena et al. 2006).

- The applied strain also seems to have been modified with figures ranging from 7\% (Abbas, Ali et al. 2006) to 90\% (Mørkøre and Einen 2003)

- The dimensions of the surface in contact with the sample, with numerous researchers following Bourne's (1968) lead and compress the sample with a platen, yet with others who reduce the diameter down to $1.2 \mathrm{~mm}$, which must be akin to a puncture test (Lamsal, Jung et al. 2007). 
- Sample sizes also vary $3 \mathrm{~mm}$ diameter cores cut out of pecan nuts (Ocón, et al. 1995) to slabs of restructured scallops which were moulded in $100 \mathrm{x}$ $100 \mathrm{~mm}$ slabs and then compressed with a $50 \mathrm{~mm}$ diameter cylindrical probe (Beltrán-Lugo, Maeda-Martínez et al. 2005). When researchers work with small food materials they often take multiple items and subject collections of the food to the double compression, for example Mohamed and co-workers (2005) tested 5 ( $2 \mathrm{~cm}$ long) noodles each time, or three intact rice grains (Moretti, Lee et al. 2005) or individual cooked rice grains with multiple replicates (Okadome et al, 1998).

- Researchers interested in compression of gels have often undertaken lubrication to minimize sample barrelling and some reported experiments with TPA have done likewise, for example Bryant, Ustunol and Steffe (1995) working with cheddar cheese.

It is not clear why such changes have been introduced, but perhaps it relates to the capability of the texture analyser being used for the study. Clearly for the instrument to work we must have a signal (preferably) mid-range of the instruments load cell. With small food items or those with very low levels of hardness, we could increase the signal by increasing the resisting force, for example using larger contact area to compress the sample will boost this. Similarly, so as to avoid going off scale and risk overloading the instrument, samples could be reduced in size. Alternatively, the signal can be reduced by slowing the speed of compression, thus giving the sample time to relax and recover between the two bites. It should be noted, however, that Shama and Sherman (1973) and Rosenthal (2010) found that the TPA performed at too slow compression speed could not be correlated with sensory evaluation. Another way of lessening the force exerted on the load cell is to reduce applied strain .

\section{II-1. Hardness}

Hardness was originally defined as "the force necessary to attain a given deformation" (Szczesniak 1963, p387). Normally the first few bites of oral tests result in gross structural breakdown of the food. If during the first TPA compression the material fractures then there is a parallel. But if no fracture occurs then the "hardness" may tell us something about the nature of the material but not how it breaks down. In the original protocol the sample height was fixed and the probe had a smaller diameter than that of the sample, thus the 
contact area on the sample was a constant. Adoption of the Instron universal testing machine made the peak shapes less pointed (Bourne, 1968). Yet the hardness is still defined as the peak force resulting from the first uniaxial compression, but since the force depends on the sample size and therefore the contact area, it would be better if the hardness were defined as the peak stress. While the original TPA used plungers of fixed cross section area, more recently researchers have used platens in which the size of the sample makes a difference. Furthermore, the stresses cannot be defined so easily for an irregular shaped food such as peas, nuts and grains. For such samples, the shape and size and how the sample is fixed on the base of the uniaxial compression apparatus as well as the compression speed should be described in detail so that the readers can repeat the measurement under the same conditions. Needless to say, the temperature and the humidity should be controlled or at least be recorded especially for samples affected by these parameters.

Although the sensorily evaluated hardness is expected to be highly correlated with the instrumental measurement, it is necessary to take into account the achievements of fracture mechanics as stressed by Peleg (2019) and Vincent (2004). Even if the sensory evaluation of foods has some specific complexities as influenced by cross modal interaction as well as being in a non-equilibrium state (Nishinari and Fang, 2018), and while foods are very complex consisting of multiple ingredients, the mechanical properties of foods can be measured objectively. Peleg (2019) and Vincent (2004) emphasize the importance of what we can learn from solid mechanics applied in material science.

Vincent, Saunders and Beyts (2002) using the critical stress intensity $K_{\mathrm{IC}}$, defined in fracture mechanics, examined the "hardness" and found a high correlation between $K_{\text {Ic }}$ and hardness for crisp foods such as carrot, apple, celery and cucumber. They found that the crispness was not correlated with $K_{\mathrm{IC}}$, and hardness and crunchiness were indistinguishable (Vincent, 2004). Rojo and Vincent (2009) studied the relation between the sensory crispness of potato crisps (commonly called chips outside UK) and the objective mechanical measurements based on stress and strain curves analysed by Weibull model. They found that crisper crisps break at a higher force, and breakdown speed of the crisp during chewing is proportional to the Weibull modulus, which is determined from the slope of the double logarithmic plot of the survival probability (= 1- failure probability) and the stress (Rojo and Vincent, 2008).

As for the structure breakdown in the mastication, the hardness has been a commonly measured variable. In most papers, hardness is represented by the 
unit of force $(\mathrm{N})$ though sometimes gravity is ignored and the mass $(\mathrm{kg})$ is inappropriately reported. Even when the contact area is reported, the size and shape of the food sample and the probe (plunger) must be stated. For example, the hardness which is often defined as the peak force of a force-deformation curve evidently depends on the size of the food sample. In the compression measurement of cylindrical watermelon with a constant height $10 \mathrm{~mm}$, Peleg (2019) shows the peak force increases with increasing diameter from $13 \mathrm{~mm}$ to $22 \mathrm{~mm}$. Kohyama and Nishinari (1992) also showed a similar behavior for cylindrical tofu with a fixed height. It might be thought impossible to record the cross sectional area of the food samples such as rice grains, peas or nuts. In some countries, such as Japan, it is common to cook rice and soybeans together and it may be necessary to evaluate the hardness of each. But, if the report does not include dimensional information for them, it is evident that the hardness values represented by the force for rice and soybean cannot be compared because their sizes and shapes are so different! Unfortunately such simple detail is often missing in many publications.

To determine the hardness accurately lubrication between the sample surface and the plunger should be taken into account. In the case of watery samples such as food gels, a small amount of water exudes from the surface and acts as a lubricant, thus the barreling of the sample is prevented. To avoid such complications, a plunger with a diameter smaller than the sample is sometimes used (Chen et al, 2013). However, in this case, the friction between the sample and the side surface of the plunger contributes to the recorded force. When, the sample shows an obvious fracture, the force shows a peak, but if no fracture occurs, the force recorded at predetermined deformation is sometimes employed as the hardness. It should not be forgotten to note the deformation in such a case.

\section{Figure 3}

Figure 3 shows a typical (single bite) puncture test of a carrot root (Horiuchi, Nishinari, Niikura and Hakamada, 1976). Arranged from the surface to the centre of the tissues are: epidermis, cortex, phloem, cambium, xylem and pith. To study the detailed mechanical property of each tissue a thin needle $(2-4 \mathrm{~mm}$ dia) was driven into the carrot (Horiuchi, et al., 1976). The force increases when the plunger is pushed into the carrot, and shows a peak force at the first firm tissue (cortex) followed by a minimum at the softer cambium, then there are further increases producing a second peak. After reaching the central tissue (pith), the plunger is withdrawn (thus the deformation is decreased), therefore the direction 
of force exerted on the plunger becomes negative. To identify the tissue at peak forces and the minimum force, it is necessary to check the distance from the surface to each tissue using a thin ( $2 \mathrm{~mm}$ dia) needle. In the case of TPA the $\mathrm{x}$ axis is taken as the time, though in terms of stress and strain it might have a clearer meaning if the $\mathrm{x}$-axis is redrawn as deformation. Such a representation is shown in Figure 3, the area under the curve and above $x$-axis represents the energy or work done by compression. The area above the curve and below the axis represents the energy or work done by decompression. Clearly the shape of the curve depends on the firmness of the different tissues.

Despite all that has been said above, the hardness is probably the most reliable TPA parameter. Bourne (2002) and Peleg (2006) explain the pitfalls from choosing the wrong geometry, size and shape. Experimenters should carefully choose the right geometry so that the deformation should occur in the intended way: when the puncture test is done, the diameter of the plunger should be less than one-third of the diameter of the sample so that the semi-infinite geometry is kept; when a sample on the support base with a hole is punctured, the diameter of the hole should not be too large to avoid the bending of the sample, and not too small to avoid the "puncture and die" in which a cylinder of the sample food is cut out from the food and pushed into the hole (Bourne, 2002).

A second and equally important point is the speed of compression. TPA is usually thought to be imitative of human mastication, and therefore the compression speed should be closer to the chewing speed of humans. Bourne (2002) showed an example of Sherman's experiment on cheese (Shama and Sherman, 1973) emphasizing the importance of compression speed. A good correlation between the hardness evaluated by sensory panel and the instrumentally measured values was only found at a certain range of compression speed. Nishinari and Fang (2018) recently discuss the effect of compression speed on the fracture stress of foods and the relation of mastication speed and the hardness. Using model starch gels, Rosenthal (2010) changed the compression speed from $0.1 \mathrm{~mm} / \mathrm{s}$ to 10 $\mathrm{mm} / \mathrm{s}$ and found the TPA hardness increased with increasing speed of compression upto $2 \mathrm{~mm} / \mathrm{s}$ and that hardness values leveled off above this speed. He recommended to choose compression speeds in excess of $2 \mathrm{~mm} / \mathrm{s}$ for TPA, yet such speeds would be totally inappropriate to measure fundamental properties such as modulus. The uniaxial compression test of gels at a lower speed is useful to get the insight of the gel structure (Nakamura, Shinoda and Tokita, 2001), but the slow compression does not correspond to the human mastication, and therefore TPA parameters obtained by such a slow compression cannot be 
compared with sensory evaluation.

Since soft foods are not bitten by teeth but squeezed or compressed between the tongue and hard palate (Arai and Yamada, 1993), for such materials perhaps the hard metal base plate of the texture analyzer could be replaced with a soft material mimicking the tongue, discriminating between deformation of the sample and the 'tongue' then become possible through videographic observation. Ishihara et al $(2013,2014)$ used an artificial tongue to simulate this situation. The force and the deformation at fracture in such an experiment is different from that obtained by a compression between two hard plates (metal or Teflon etc). This difference can be predicted from the fracture probability of model gels and the proportion of subjects who decide to squeeze the food between tongue/palate.

\section{II-2. Adhesiveness}

In TPA adhesiveness was originally defined as "the work necessary to overcome the attractive forces between the surface of the food and the surface of other materials with which the food comes in contact (e.g., tongue, teeth, palate, etc.)" (Szczesniak 1963, p387). Adhesiveness is not exhibited by all foods. When TPA curves show multiple breaks (brittleness/fracturability) during the first compression generally adhesiveness is absent, yet when present it shows up as a negative peak after the first compression. Traditionally, adhesiveness was determined as the area under the negative force - deformation curve, i.e. the energy required to raise and separate the plunger from the food sticking to it.

The adhesiveness can be quantified by the negative peak force or the energy which is exerted on the plunger pulled apart from the sample. If the sample does not stick to the plunger at all, the adhesiveness is estimated as zero.

Adhesiveness is related to the ability of the sample to wet the surface and when the surfaces are pulled apart the material or liquid at the surface must flow into the gap created by the separating surfaces. Adhesiveness is caused by the force between the food sample and the plunger imitating the role of teeth, tongue or palate. When a puncture test geometry is used and the probe penetrates the surface of the food, e.g. raw carrot in Figure 3, the adhesiveness should be evaluated cautiously. In such a case, the negative peak force area is driven by the carrot tissues which surrounded the penetrating plunger and not any inherent stickiness. An obvious and different situation exists if we imagine a similar 
penetration test on sticky rice cake. The origin of the exerted force from the sample is different, and therefore the negative peak in TPA cannot always be thought as having originated from adhesiveness.

Nishinari and Fang (2018) show that even if two samples have the same TPA adhesiveness, the sticky sensation may be different if the force curves are tall \& narrow as opposed to short $\&$ broad as shown in Figure 4 . The attached part of a food to the plunger is separated at a short distance in the former case while the latter curve shows a long distance though the force required is smaller. Another important point for the adhesiveness is that this value depends strongly on the brittleness or crumbliness of the food. The area of compressed food sticking to the plunger is different among foods as shown in Brenner and Nishinari (2014). Therefore, for crumbly solid foods, the adhesiveness should better be measured at a relatively small compression before the sample fractures so that the contact area does not change at the first contact in the compression (plunger downward) and in the extension (plunger upward).

\section{Figure 4}

In an oral situation it should be remembered that the chewed food with incorporated saliva must be compressed to a certain extent for it to show any adhesiveness.

It might be useful to compare the two cases: in a liquid-like food and a solid-like food. In the former case, the liquid sample sticking to the side and the bottom surfaces of the plunger will exert the downward force when the plunger is raised up. In the latter case, the excluded part of compressed or penetrated food tend to recover by virtue of the elasticity, and thus exert the negative force when the plunger is raised up. Even though the direction of the force exerted by food to the plunger is similarly negative, the origin of the force is different. Humans maybe able to distinguish this difference, while the instrumental TPA cannot tell the difference because it detects only the force and deformation. In addition, the liquid flows in the oral cavity and thus sensed not only by teeth but also by the tongue and hard palate while the solid may be sensed mostly by the teeth in the first bite. Therefore, we should be cautious in interpreting the meaning of TPA adhesiveness.

Instrumentally measured adhesiveness has been reported to be well correlated with sensory evaluation where the comminuted food stick to the oral organs, the tongue, palate and teeth. Since the boli are disintegrated and mixed with saliva, 
the adhesiveness may be reasonably well characterized by TPA for such foods. In most published papers, the adhesiveness of bread was reported as zero. It may be true if the bread is compressed without adding saliva. Since the TPA is an imitation measurement, the addition of saliva and temperature control are necessary to evaluate the adhesiveness of bread. The addition of saliva will of course affect the textural characteristics of most foods.

In Japan, palatability of cooked rice is determined by texture, and this is understood by the fact that cooked rice has only a weak taste and aroma intensity (which is different from scented rice preferred in Thailand). This is also true for other staple foods like noodles, bread, and potatoes which are consumed in great quantities as an energy source. The preference of cooked rice depends on food culture and history. For example, in France rice grains which stick each other are not liked while in Japan appropriate stickiness is the most required characteristics not only for sushi or rice balls but also for cooked rice. Two methods to evaluate the textural characteristics of cooked rice have been used, 1 ) cooked rice in a cup is compressed by a plunger, 2) one or a few cooked rice grains are separately put on the base of a uniaxial compression apparatus, and then compressed by a plunger. Matsuo and co-workers (2002) using the first method, found that the combination of stickiness (the maximum force of the negative peak in the uniaxial compression test in Figure 4) and adhesiveness (the area enclosed by the negative peak and the baseline $A_{3}$ in Figure 4) are highly correlated with preference. The preference in the sensory evaluation has been known highly correlated with sensory stickiness and adhesiveness. They used three cylindrical ( $12 \mathrm{~mm}$ diameter) plungers in the uniaxial compression to get average values for the rice grains cooked in a metal cup without moving cooked rice sample to the plate of the apparatus to avoid the change in densification. It was more effective to use both the energy $A_{3}$ and the peak force $F_{3}$ to correlate with the sticky and adhesive sensation. Okadome and colleagues (1998) using the second method they performed a uniaxial compression, at compression ratios $25 \%, 90 \%$ as well as a continuous progressive compression test using both one cooked rice grain and an average for 10 grains. They found that low compression tests were useful to correlate the instrumental data with sensory stickiness which might be determined by stickiness of surface layer of cooked rice grain. Recently, Yu et al (2019) employing compression at small deformation range for individual rice grains and using a shear ring tester based on Schulze's method (described in the next section) demonstrated a possibility of further development of understanding the cooked rice texture. 
In the oral cavity, the adhesive force between food fragments and the oral organs (teeth, tongue and palate) was evaluated. In the instrumental measurement, the adhesive force between food and the plunger was evaluated, but since the surface properties of plunger affect the detected force, it might be simple to measure the adhesive force between the fragments of the same food.

Adhesiveness may be evaluated by compressing a food sample glued to the base of a texture analyzer by another food sample of the same size and shape glued on the plunger. The deformation should not be too large so that the contact area is well defined. Nitta et al (2018) evaluated the adhesiveness of noodle strand by this method.

\section{II-3. Cohesiveness}

Szczesniak (1963 p387) defined cohesiveness "as the strength of the internal bonds making up the body of the product", a definition that works well in the sensory evaluation of foods. In the context of the instrumental TPA, Friedman and colleagues (1963, p393) explain that cohesiveness is "a direct function of the work needed to overcome the internal bonds of the material." These authors went on to attribute it to the ratio of the areas under the twin peaks. Drake cited by Breene (1975) expressed concern as to the appropriateness of naming the ratio of the two peaks as cohesiveness. The ratio of the area under the peaks is a meaningful measure, but probably one that relates to recovery after deformation. In his attempt to relate outcomes from TPA to well defined rheological parameters, Sherman (1969) points out that cohesion (and hardness) relate to elasticity. Despite being recognized as a primary parameter from the TPA, the General Foods scientists were not able to identify foods that provided varying degrees of cohesiveness when attempting to create standard rating scales (Szczesniak, Brandt and Friedman, 1963). If cohesiveness is the strength of the internal bonds then we would need to measure it by pulling the material apart in all directions and hence its units should be $\mathrm{Nm}^{-2}$ indicating a similar property to the bulk modulus (in expansion). While uniaxial deformation, has been widely used in compression and extension to study rubber-like materials (Treloar, 1975), the negative hydrostatic pressure test has rarely been employed because of the difficulty in the instrumentation. Biaxial extension is sometimes used to study rubber-like materials and gels (Urayama, Kawamura and Kohjiya, 2009). Since biaxial data can offer some information which is not available by uniaxial extension, the three dimensional expansion experiments on foods would be useful to get more insight on the cohesiveness. It is worth noting that the bulk modulus (like the elongational elastic modulus) should be the same whether measured in 
compression or expansion (extension in the case of the elongational elastic modulus). Thus we may be able to estimate the cohesiveness in a compressive mode.

In view of the comments above, the value $A_{2} / A_{1}$ from the TPA curve should not be called cohesiveness and should not be defined as "the strength of the internal bonds making up the body of the product", but should perhaps be referred to as "structural recoverability" (Nishinari and Fang, 2018). Mioche and Peyron (1995) studied the bite force for model foods with elastic, plastic and brittle textures (silicone elastomers, waxes and pharmaceutical tablets, etc) at $10 \%$ strain. They found that elastic model foods showed almost complete recovery in spite of a slight hysteresis, while a plastic model food showed a large hysteresis because of the large unrecoverable deformation. When viscous and plastic components coexist in a model food, the force-deformation curve shows the hysteresis in the compression and decompression by a plunger. The recoverability parameter $\left(A_{2} / A_{1}\right)$ could be considered in a similar way to adhesiveness (Figure 4$)$. That is not only the ratio of the area enclosed by the force-deformation curve but also a comparison of the peak forces of different curves.

Another approach might be to measure cohesiveness of solid foods by methods widely used in solid mechanics. The cohesiveness of solid particles has been estimated by flowability defined as the ratio $\mathrm{ff}_{\mathrm{c}}$ of unconfined yield strength $\sigma$ u to the consolidation stress $\sigma_{\mathrm{c}}\left(f f_{\mathrm{c}}=\sigma_{\mathrm{c}} / \sigma_{\mathrm{u}}\right)$. Here, the unconfined yield strength is the minimum normal stress required to cause the yield of a cylindrical solid, while the consolidation stress is the normal stress on the same cylinder confined in a strong hollow metal cylinder (Figure 5). The unconfined yield strength is an increasing function of the consolidation stress (Schulze, 2008). The unconfined yield strength increases with increasing consolidation stress. The larger value of $f f_{c}$ indicates the higher flowability of the material in that conditioned densification. The flow behavior of solid particles is classified by the value of the flowability $f f_{c}$, as: $f f_{c}<1$ (not flowing), $1<f f_{c}<2$ (very cohesive), $2<f f_{c}<4$ (cohesive), $4<$ $f f_{c}<10$ (easy-flowing), $10<f f_{c}$ (free-flowing) (Scheltze, 2008). See also a review on the cohesiveness and flowability of powders by Peleg (1977). This method first proposed in powder evaluation by Jenike (1964) was developed by Ashton, Cheng, Farley and Valentin, 1965) and Schulze (2008), and used widely for granular materials. Recently, ring shear test based on this method has been applied to food samples such as pea powders with different moisture contents (Tobin et al , 2017) and cooked rice (Yu et al, 2019). These papers show great potential for the ring shear test for wider range of food samples. 


\section{Figure 5}

The above approach to evaluate cohesiveness of solid foods may be suitable for fragmented foods, but less so for non-fragmented foods. In the case of solid foods, the size distribution of fragments after repeated compression may be closely related with the cohesiveness. Non-cohesive foods and brittle foods may be crushed into smaller fragments. Kobayashi and his coworkers analyzed the particle size distribution after mastication (Kobayashi, Kohyama, Sasaki, and Matsushita, 2006; Kobayashi, Kohyama, Shiozawa, 2010; Moritaka, Yamanaka, Kobayashi, Ishinhara, Nishinari, 2019). Although they did not use the word cohesiveness in the sense "as the strength of the internal bonds making up the body of the product", it seems that the size distribution reflects this property.

Peleg (1976) proposed a corrected shape of a TPA curve obtained as shown in Figure 6 . This has similarities to Figure 3 in that the $x$-axis is not the time but the deformation (distance). He pointed out that the $x$-axis of the original TPA should be converted to the distance from the time so that the area under the curve has the meaning of the energy or the work done during the compression. We should pay attention that the distance in TPA is taken downward from the time of the contact of the plunger with the food sample. After compression to a predetermined deformation, the plunger is raised up so that the displacement is decreased as shown in Figures 3 and 6.

\section{Figure 6}

It is self-evident that the forces shown in Figure 6 depends on the nature of food and whether they stick to the plunger or not. Semi-solid type yogurt or rice cakes stick to the plunger and this contributes to the force detected. The force exerts from the lower to upper direction to the plunger. The adhered portion of the sample exerts a negative force on the plunger acting downwards.

TPA is intended for solid foods, yet viscoelasticity is a continuum and deciding at what consistency the test is no longer valid is a value judgement. Nevertheless, when the food is a liquid, the application of TPA is both inappropriate and disastrous! Nishinari et al (2013) have clearly shown that the so-called cohesiveness of water is 1 and decreases with increasing concentration of xanthan gum. The reason is clear, water recovers very fast the initial horizontal 
plane during the upstroke of the plunger and thus the second bite records the same force value as in the first bite. Concentrated xanthan gum solution sticks to the plunger and the initial horizontal plane cannot be recovered completely before the second stroke commences, and in addition the recovery of the structure takes a time, and thus the thixotropic nature should be taken into account (Barnes, 1997). This tendency is increased with increasing concentration of xanthan. Therefore, the so-called cohesiveness determined by a conventional TPA is decreased with increasing concentration of xanthan. Imagine a semi-solid yogurt used for dysphagic patients instead of xanthan, the surface of yogurt does not recover its original shape before the second bite. This shows clearly that a conventional TPA cohesiveness is meaningless for fluids. To quantify the cohesiveness of liquids, it is recommended that we estimate the force and deformation of the liquid when it is subjected to an extension experiment, taking into account the rate dependence, or estimate the length of liquid filament flowing down from a narrow tube (Nishinari, Turcanu, Nakauma, Fang, 2019).

There is a sense that having defined the notion of cohesiveness from a sensory point of view the creators of TPA desired to include it in their instrumental technique. Yet with no obvious way to measure the strength of the internal bonds, the authors conceived the ratio of the positive peaks as something that might be related, and shoehorned this disparate definition and the measurement together. Apart from poorly matched measurement and definition, it is worrying that the so-called cohesiveness is used in two of the derived terms: gumminess and chewiness.

The common use of TPA to evaluate adhesiveness and cohesiveness is flawed, as the physical properties of an intact specimen only provide very limited insights to these attributes. This is because these properties depend on many factors including granulation and hydration that are not emulated under TPA conditions as mentioned earlier. Addition of artificial saliva or water for the adhesiveness evaluation may improve the TPA procedure, but it also depends on the degree of deformation and then the fragmentation complicates the situation such as the uncertainty of the contact area of the probe (plunger) and the food sample.

\section{Conclusion}

TPA has been used widely and will be used because it is easy to use. However, the parameters obtained should be cautiously interpreted. To avoid common 
misuses of TPA parameters the following should be taken into account: Hardness should be measured at higher compression speed. Temperature dependent samples such as gelatin gels and fat should be measured in temperature controlled condition. The size and shape of the samples and plunger should be described in figure captions because often figures are cited by other researchers without including measurement conditions. Adhesiveness should be measured separately at a lower compression ratios so that the area of contact with the plunger does not change especially for brittle or crumbly food samples. For dried samples such as biscuits and bread, it is recommended to add artificial saliva or water to evaluate adhesiveness because without fluids the adhesiveness of these foods are to be estimated as zero, which does not reflect the reality. In the context of TPA the term cohesiveness (originally defined as $A_{2} / A_{1}$ ) should not be used, but replaced by recoverability (and perhaps Peleg's corrected cohesiveness should be named corrected recoverability) but this should be further discussed in the texture community. This recoverability should not be used for liquid samples. The true cohesiveness of foods should be quantified by other measurements such as fluid extension for liquids, and flowability index or bulk modulus for solid foods. The cohesiveness of bolus should be carefully estimated because it is sometimes very close to liquid.

The words we use to describe the consistency of foods during instrumental processing and oral processing should tally, yet in this paper we have questioned the meaning of the parameters defined in TPA. Consequently our understanding of the "cohesive" bolus formed in oral processing may not tally with "cohesiveness" as defined in TPA. While brittleness (fracturability) named in TPA has a parallel to structural breakdown in the mouth - hardness without fracture is probably irrelevant to oral processing. We have tried to look critically at both the oral trajectory of a range of foods, some which follow the widely accepted breakdown path and some which appear not to fit that model; and instrumental measures of corresponding textural properties.

\section{Ethical statement}

The authors declare that they do not have any conflict of interest. This study does not involve any human or animal testing.

\section{References}


Abbas, S. A., Ali, S., Halim, S. I. M., Fakhrul-Razi, A., Yunus, R. and Choong, T. S. Y. (2006). Effect of thermal softening on the textural properties of palm oil fruitlets. J. Food Eng., 76, 626-631.

Allais, I., R.-B. Edoura-Gaena and É. Dufour (2006). Characterization of lady finger batters and biscuits by fluorescence spectroscopy-Relation with density, color and texture. J. Food Eng., 77, 896-909.

Arai, E. and Yamada, Y. (1993). Effect of the texture of food on the masticatory process. Jpn J. Oral Biol., 35, 312-322.

Ashton, M. D., Cheng, D. C. H., Farley, R. and Valentin, F. H. H. (1965). Some investigations into the strength and flow properties of powders. Rheol. Acta, $\mathbf{4}$, 206-218.

Barnes, H.A. (1997). Thixotropy a review, J. Non-Newtonian Fluid Mech., 70, 133.

Beltrán-Lugo, A. I., A. N. Maeda-Martínez, R. Pacheco-Aguilar, H. G. NolascoSoria and V. M. Ocaño-Higuera (2005). Physical, Textural, and Microstructural Properties of Restructured Adductor Muscles of 2 Scallop Species Using 2 Coldbinding Systems. J. Food Sci., 70, E78-E84.

Bourne, M. C. (1968). Texture profile of ripening pears. J. Food Sci., 33, 223226.

Bourne, M. C. (2000). Why so many tests to measure texture? In Nishinari, K. Ed. Hydrocolloids-Part2, Fundamentals and Applications in Food, Biology, and Medicine, Elsevier, pp.425-430.

Bourne, M. C. (2002). Food texture and viscosity (2nd ed.). New York, NY: Academic Press.

Breene, W. M. (1975). Application of Texture Profile Analysis to Instrumental Food Texture Evaluation. J. Texture Stud., 6, 53-82.

Brenner, T. and Nishinari, K. (2014). A note on instrumental measures of adhesiveness and their correlation with sensory perception. J. Texture Stud., 45, 74-79.

Brody, A. L. (1957). Masticatory Properties of Foods by the Strain Gauge Denture 
Tenderometer. PhD Thesis, Massachusetts Institute of Technology.

Bryant, A., Z. Ustunol and J. Steffe (1995). Texture of Cheddar Cheese as Influenced by Fat Reduction. J. Food Sci., 60, 1216-1219.

Chen, J. (2009). Food oral processing- a review, Food Hydrocoll. 23, 1-25.

Chen, J. and Engelen, L. (Eds.). (2012). Food oral processing: Fundamentals of eating and sensory perception. Chichester, UK: Wiley-Blackwell.

Chen, J., Khandelwal, N., Liu, Z. and Funami, T. (2013). Influences of food hardness on the particle size distribution of food boluses. Arch Oral Biol, 58, 293-298.

Chen, J. and Loret, C. (2017). The $4^{\text {th }}$ International conference on food oral processing, July 2016. J. Texture Stud. 48, 269-270.

Chen, J.; Lolivert, L. (2011). The determining role of bolus rheology in triggering a swallowing. Food Hydrocoll. 25, 325-332.

Chen, J. and Stokes, J. R. (2012). Rheology and tribology: two distinctive regimes of food texture sensation. Trends Food Sci. Technol. 25, 4-12.

Chojnicka-Paszun, A., de Jongh, H. H. J. and de Kruif, C. G. (2012). Sensory perception and lubrication properties of milk: Influence of fat content. Int. Dairy J. 26, 15-22.

Dresselhuis DM, de Hoog EHA, Cohen Stuart MA, van Aken GA. (2008). Application of oral tissue in tribological measurements in an emulsion perception context. Food Hydrocoll. 22, 323-335.

Engelen L, van der Bilt A., Schipper M. and Bosman F. (2005a). Oral size perception of particles: effect of size, type, viscosity and method. J. Texture Stud. 36, 373-86.

Engelen, L., Fontijn-Tekamp, A. and van der Bilt, A. (2005b). The influence of product and oral characteristics on swallowing. Arch. Oral Biol., 50, 739-746.

Fiszman, S. and Tarrega, A. (2018). The dynamics of texture perception of hard solid food: A review of the contribution of the temporal dominance of sensations technique. J. Texture Stud. 49, 202-212.

Friedman, H. H., J. E. Whitney and A. S. Szczesniak (1963). The Texturometer-A 
new instrument for objective texture measurement. J. Food Sci., 28, 390-396.

Gray-Stuart, E.M. Jones, J. R. Bronlund, J. E. (2017). Defining the end-point of mastication. J Texture Stud., 48, 345-356.

Hawthornthwaite, D., Y. Ramjan and A. Rosenthal (2015). Oral processing of low water content foods - A development to Hutchings and Lillford's breakdown path. J. Texture Stud., 46, 212-218.

Hiiemae, K. (2004). Mechanisms of food reduction, transport and deglutition: How the texture of food affects feeding behavior. J. Texture Stud. 35, 171-200.

Horiuchi, H., Nishinari, K., Niikura, M. and Hakamada, K. (1976). Effect of freezing process on the texture of vegetables. Part II. Measurement of the puncture curves of carrot in freezing process. Nippon Shokuhin Kogyou Gakkaishi, 23, 468-473. (In Japanese with English summary and figure captions)

Hutchings, J. B., \& Lillford, P. L. (1988). The perception of food texture-The philosophy of the breakdown path. J. Texture Stud., 9, 103-115.

Ishihara, S., Isono, M., Nakao, S., Nakauma, M., Funami, T., Hori, K., et al (2014). Instrumental uniaxial compression test of gellan gels of various mechanical properties using artificial tongue and its comparison with human oral strategy for the first size reduction. J. Texture Stud., 45, 354-366.

Ishihara, S., Nakao, S., Nakauma, M., Funami, T., Hori, K., Ono, T., et al. (2013). Compression test of food gels on artificial tongue and its comparison with human test. J. Texture Stud., 44, 104-114.

Jalabert-Malbos, M. L., Mishellany-Dutour, A., Woda, A., and Peyron, M. A. (2007). Particle size distribution in the food bolus after mastication of natural foods. Food Qual Prefer. 18, 803-812.

Jenike, A. W. (1964). Storage and flow of solids. Bull. of the University of Utah, 53, 26; Bull No. 123 of the Utah Engineering Experiment Station https://digital.library.unt.edu/ark:/67531/metadc1067072/m2/1/high res d/5 240257.pdf

Juarez-Enriquez, E., Olivas, G. I., Zamudio-Flores, P. B., Ortega-Rivas, E., PerezVega, S., and Sepulveda, D. R. (2017). Effect of water content on the flowability of hygroscopic powders, J. Food Eng., 205, 12-17. 
Kobayashi, N., Kohyama, K., Sasaki, Y. and Matsushita, M. (2006): Statistical Laws for Food Fragmentation by Human Mastication. J. Phys. Soc. Jpn. 75, 083001.

Kobayashi, N., Kohyama, K. and Shiozawa, K. (2010). Fragmentation of a Viscoelastic Food by Human Mastication. J. Phys. Soc. Jpn. 79, 044801.

Kohyama, K. and Nishinari, K. (1992). Some Problems in Measurements of Mechanical Properties of Tofu (Soybean Curd). Nippon Shokuhin Kogyo Gakkaishi, 39, 715-721(In Japanese with English summary).

Lamsal, B. P., Jung, S. and Johnson, L. A. (2007). "Rheological properties of soy protein hydrolysates obtained from limited enzymatic hydrolysis." LWT - Food Sci. Technol. 40, 1215-1223.

Matsuo M., Takaya T., Miwa S., Moritaka H., and Nishinari K. (2002). The uniaxial compression test as a simulation of mastication for the texture evaluation of cooked rice, Nippon Soshaku Gakkaishi (Jap Soc Mastication Health Promotion). 12, 11-25. (In Japanese with English summary).

Mioche, L. and Peyron, M. A. (1995). Bite force displayed during assessment of hardness in various texture contexts. Arch. Oral Biol. 40, 415-423.

Mohamed, A. A., P. Rayas-Duarte, J. Xu, D. E. Palmquist and G. E. Inglett (2005). Hard red winter wheat/nutrim-OB alkaline fresh noodles: Processing and texture analysis. J. Food Sci., 70, S1-S7.

Moretti, D., T.-C. Lee, M. B. Zimmermann, J. Nuessli and R. F. Hurrell (2005). "Development and Evaluation of Iron-fortified Extruded Rice Grains." J. Food Sci., 70, S330-S336.

Moritaka,H., Yamanaka, K., Kobayashi, N., Ishihara, M. and Nishinari, K. (2019). Effects of the gel size before ingestion and agarose molecular weight on the textural properties of a gel bolus. Food Hydrocoll., 89, 892-900.

Mørkøre, T. and O. Einen (2003). Relating Sensory and Instrumental Texture Analyses of Atlantic Salmon. J. Food Sci., 68, 1492-1497.

Nishinari, K. and Fang, Y. (2018). Perception and measurement of food texture: Solid foods. J. Texture Stud., 49, 160-201.

Nishinari, K. and Fukada, E. (1980). Viscoelastic, dielectric and piezoelectric 
behaviour of solid amylose. J.Polym. Sci. Polym. Phys. Ed., 18, 1609-1619.

Nishinari, K., Kohyama, K., Kumagai, H., Funami, T. and Bourne, M. (2013).

Parameters of texture profile analysis. Food Sci Technol Res, 19, 519-521.

Nishinari, K., Turcanu, M., Nakauma, M. and Fang, Y. (2019). Role of the viscosity and cohesiveness in safe swallowing, npj Sci Food, 3 (1): 5.

http://www.nature.com/articles/s41538-019-0038-8

Nitta, Y. Yoshimura, Y., Ganeko, N., Ito, H., Okushima, N., Kitagawa, M. et al. (2018). Utilization of Ca2+-induced setting of alginate or low methoxyl pectin for noodle production of Japonica rice. LWT - Food Sci Technol, 97, 362-369.

Ocón, A., Anzaldúa-Morales, A., Quintero, A. and Gastélum, G. (1995). Texture of pecans measured by sensory and instrumental means. J.Food Sci., 60, 13331336.

Okadome, H., Toyoshima, H., Suto, M., Ando, I., Numaguchi, K., Horisue, N. and Ohtsubo, K. (1998). Palatability evaluation for Japonica rice grains based on multiple physical measurements of individual cooked rice grain, Nippon Shokuhin Kagaku Kogaku Kaishi, 45, 398-407.

Olthoff L. W., van der Bilt A., Bosman, F. and Kleizen H. H. (1984). Distribution of particle sizes in food comminute by human mastication. Arch Oral Biol., 29, 899-903.

Peleg, M. (1976). Texture profile analysis parameters obtained by an Instron universal testing machine. J. Food Sci., 41, 721-722.

Peleg, M. (1977). Flowability of food powders and methods for its evaluation: A review," J. Food Process Eng., 1, 303-328.

Peleg, M. (2006). On fundamental issues in texture evaluation and texturizationA view. Food Hydrocoll., 20, 405-414.

Peleg, M. (2019). The instrumental texture profile analysis revisited. J. Texture Stud., 50, 1-7. https://doi.org/10.1111/jtxs.12392

Pereira, L. J., de Wijk, R. A., Gavião, M. B. D., and van der Bilt, A. (2006). Effects of added fluids on the perception of solid food. Physiol. Behav. 88, 538-544.

Pereira, L. J., Gavião, M. B. D., Engelen, L. and van der Bilt, A. (2007). Mastication and swallowing: Influence of fluid addition to foods. J. App/ Oral 
Sci., 15, 55-60.

Peyron, M. A., and Woda, A. (2016). An update about artificial mastication. Curr. Opin. Food Sci., 9, 21-28.

Prinz, J. F. and Lucas, P. W. (1997). Mastication and swallowing: an optimization model. Proc. Royal Soc. London ser. B 264, 1715-1721.

Proctor, B. E., S. Davison and A. L. Brody. (1955). A recording strain-gauge denture tenderometer for foods. I. Instrument evaluation and initial tests. Food Technol. 9, 471.

Ravi, R. and N. S. Susheelamma (2004). "The effect of the concentration of batter made from Chickpea (Cicer arietinum L.) flour on the quality of a deepfried snack." Int. J. Food Sci. \& Technol., 39, 755-762.

Rojo, F.J., Vincent, J. F. V. (2008). Fracture properties of potato crisps. Int. J. Food Sci. Technol., 43, 752-760.

Rojo, F. J. and Vincent, J. F. V. (2009). Objective and subjective measurement of the crispness of crisps from four potato varieties. Engineering Failure Analysis, 16,2698-2704

Rosenthal, A. J. (2010). Texture profile analysis: How important are the parameters? J. Texture Stud., 41, 672-684.

Rosenthal, A. J. and A. Pang (2018). "Oral Processing of Crackers: Changes in the Secondary Textural Characteristics." J. Nutrition, Food and Lipid Sci. 1, 1-5.

Rosenthal, A.J. and Share, C. (2014). Temporal dominance of sensations of peanuts and peanut products in relation to Hutchings and Lillford's "breakdown path". Food Qual. Prefer. 32 (C), 311-316.

Rosenthal, A.J. and Yilmaz, S. (2015). Possible Mechanism behind the Hard-toSwallow Property of Oil Seed Pastes. Int. J. Food Prop., 18, 2077-2084.

Shama, F., and Sherman. P. (1973). Evaluation of some textural properties of foods with the Instron Universal Testing Machine. J. Texture Stud., 4, 344-353.

Sherman, P. (1969). "A Texture Profile of Foodstuffs Based upon Well-defined Rheological Properties." J. Food Sci., 34, 458-462. 
Schulze, D. (2008). Powders and bulk solids (Springer Verlag Berlin, 2008).

Stokes J. R. (2012). 'Oral' rheology. In: Chen J, Engelen L, editors. Food oral processing: fundamentals of eating and sensory perception. Wiley Blackwell, pp. 227-263.

Stokes, J. R., Boehm, M. W. and Baier, S. K. (2013). Oral processing, texture and mouthfeel: From rheology to tribology and beyond. Curr. Opin. Colloid Interf. Sci., 18, 349-359.

Szczesniak, A. S. (1963). Classification of textural characteristics. J. Food Sci., 28, 385-389.

Szczesniak, A. S. (2002). Texture is a sensory property. Food Qual. Prefer., 13, 215-225.

Szczesniak, A. S., Brandt, M. A. and Friedman, H. H. (1963). "Development of standard rating scales for mechanical parameters of texture and correlation between the objective and the sensory methods of texture evaluation." J. Food Sci., 28, 397-403.

Tobin, A. B., Heunemann, P., Wemmer, J., Stokes, J. R., Nicholson, T., Windhab, E. J., et al. (2017). Cohesiveness and flowability of particulated solid and semisolid food systems. Food Funct., 18, 3647-3653.

Torres, O., Andablo-Reyes, E., Murray, B. S. and Sarkar, A. (2018). Emulsion microgel particles a high-performance bio-lubricants. ACS Appl. Mater. Interfaces, 10, 26893-26905.

Treloar, L. R. G. (1975). The physics of rubber elasticity, 3rd Ed., Clarendon Press, Oxford, UK.

Tyle, P. (1993). Effect of size, shape and hardness of particles in suspension on oral texture and palatability. Acta Physiol., 84, 111-118.

Urayama, K., Kawamura, T and Kohjiya, S. (2009). Structure-mechanical property correlations of model siloxane elastomers with controlled network topology. Polymer, 50, 347-356.

van der Bilt, A., Olthoff, L.W., van der Glas, H.W., van der Weelen, K. and Bosman, F. A. (1987). Mathematical description of the comminution of food during mastication in man. Arch Oral Biol., 32, 579-586. 
van Eck , A., Hardeman, N., Karatz, N., Fogliano, V., Scholten, E. and Stieger, M. (2019). Oral processing behavior and dynamic sensory perception of composite foods: Toppings assist saliva in bolus formation. Food Qual Prefer.,71, 497-509.

Vincent, J. F. V. (2004). Application of fracture mechanics to the texture of food. Engineering Failure Analysis, 11, 695-704.

Vincent, J. F. V., Saunders, D. E. J., and Beyts, P. (2002). The use of critical stress intensity factor to quantify "hardness" and "crunchiness" objectively. $J$ Texture Stud., 33, 149-59.

Yu, L., Witt, T. Rincon Bonilla, M., Turner, M.S., Fitzgerald, M. and Stokes, J.R. (2019). New insights into cooked rice quality by measuring modulus, adhesion and cohesion at the level of an individual rice grain. J.Food Eng., 240, 21-28. 